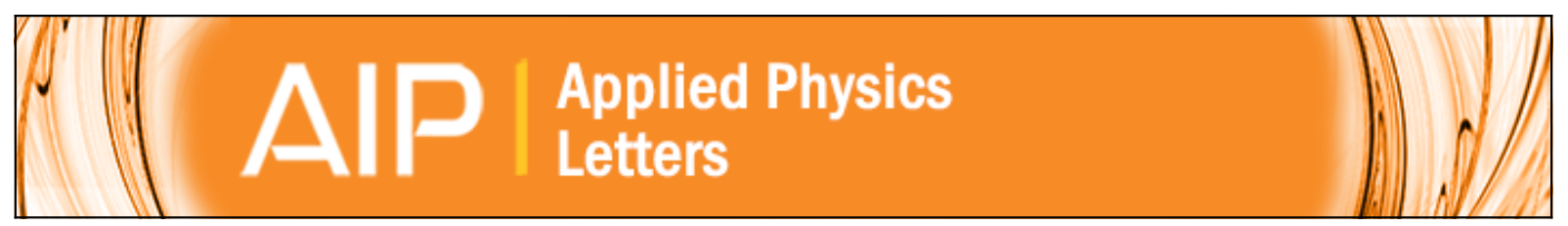

\title{
On the effect of alignment layers on blue phase liquid crystals
}

Pankaj Joshi, Xiaobing Shang, Jelle De Smet, Esma Islamai, Dieter Cuypers, Geert Van Steenberge, Sandra Van Vlierberghe, Peter Dubruel, and Herbert De Smet

Citation: Applied Physics Letters 106, 101105 (2015); doi: 10.1063/1.4914888

View online: http://dx.doi.org/10.1063/1.4914888

View Table of Contents: http://scitation.aip.org/content/aip/journal/apl/106/10?ver=pdfcov

Published by the AIP Publishing

\section{Articles you may be interested in}

Optical rotatory power of polymer-stabilized blue phase liquid crystals

Appl. Phys. Lett. 102, 131102 (2013); 10.1063/1.4799511

Fast tunable reflection in amorphous blue phase III liquid crystal

J. Appl. Phys. 113, 123103 (2013); 10.1063/1.4797492

An experimental investigation of electrically induced-birefringence of Kerr effect in polymer-stabilized blue phase liquid crystals resulting from orientations of liquid crystals

Appl. Phys. Lett. 101, 093501 (2012); 10.1063/1.4748117

Fast electro-optic switching in liquid crystal blue phase II

Appl. Phys. Lett. 98, 131905 (2011); 10.1063/1.3564896

Liquid crystal alignment with a molecular template of imprinted polymer layer during phase separation Appl. Phys. Lett. 88, 113504 (2006); 10.1063/1.2184891

Want to publish your paper in the \#1 MOST CITED journal in applied physics?

With Applied Physics Letters, you can.

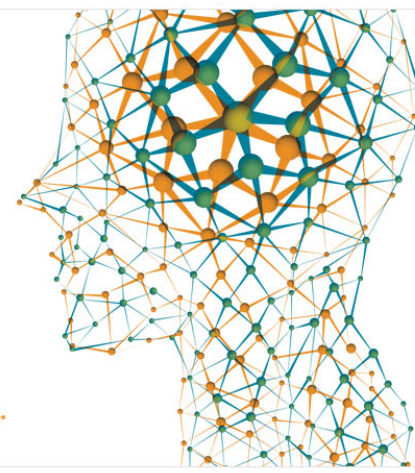




\title{
On the effect of alignment layers on blue phase liquid crystals
}

\author{
Pankaj Joshi, ${ }^{1, a)}$ Xiaobing Shang, ${ }^{1}$ Jelle De Smet, ${ }^{1}$ Esma Islamai, ${ }^{1}$ Dieter Cuypers, ${ }^{1}$ \\ Geert Van Steenberge, ${ }^{1}$ Sandra Van Vlierberghe, ${ }^{2}$ Peter Dubruel, ${ }^{2}$ and Herbert De Smet ${ }^{1}$ \\ ${ }^{1}$ Centre for Microsystems Technology (CMST), imec and Ghent University, 9000 Gent, Belgium \\ ${ }^{2}$ Polymer Chemistry and Biomaterials Group, Ghent University, 9000 Gent, Belgium
}

(Received 11 December 2014; accepted 3 March 2015; published online 11 March 2015)

\begin{abstract}
In the present work, the effect of alignment layers on blue phase liquid crystals was investigated. It was found that homogeneous alignment layers have profound selective influence on blue phase II (BPII). In the absence of alignment layers, BPII domains were randomly oriented and showed weak Bragg reflection in the UV, whereas with assistance of anchoring uniform domains with sharp Bragg reflection in the visible range appeared. On the other hand, the magnitude of Bragg shift in response to alignment layers in BPI is negligible. Domains of BP with alignment layers exhibit sharp Bragg reflection peaks (with $\mathrm{FWHM}<15 \mathrm{~nm}$ ), with very vivid colors and possessing fast switching speeds $\left(<5 \times 10^{-4} \mathrm{~s}\right)$. This simple method of selectively assisting one of the cubic phases is expected to be advantageous in the comparative studies of the two phases. (C) 2015 AIP Publishing LLC. [http://dx.doi.org/10.1063/1.4914888]
\end{abstract}

Blue phase liquid crystals (BPLC) have recently become a very interesting field of study. BPs are exotic phases exist between chiral Nematic $\left(\mathrm{N}^{*}\right)$ and isotropic phases and naturally occurring only in a very narrow temperature range in highly chiral liquid crystals. The presence of a large Kerr constant $^{1,2}$ and ultra-fast switching ${ }^{3}$ characteristics makes them especially appealing for a myriad nano-photonic components. Since in nature they exist only in a narrow temperature range of $\sim 1-2{ }^{\circ} \mathrm{C}$, the scope of their application used to be severely limited. However, since the development of the in situ polymerization technique which renders BPLCs stable over several tens of degree Celsius, ${ }^{4}$ they have quickly emerged as the candidate material for advanced liquid crystal displays. Three distinct thermodynamic blue phases are known to exist, namely, BPI, BPII, and BPIII in order of increasing temperature. BPI and BPII consist of double twisted cylinders (DTC) arranged in three dimensions, forming body-centered (bcc) and simple cubic ( $s c$ ) symmetries in their director fields, respectively. BPIII is known to be an amorphous network of disclinations. ${ }^{5}$ The lattice constant of the cube is determined by the radius of the DTC which in turn is dictated by the chiral pitch. The lattice constant of BPI is four times the diameter of the DTC (equal to the pitch), whereas for BPII it is two times the diameter of the DTC (equal to half of the pitch). They also differ both in the ratio of the unit cell size to the volume occupied by the DTCs and in the length of disclination lines present per unit cell. Owing to these fundamental differences, BPI and BPII differ in their response to external fields and in their optical properties. For example, BPII shows faster response times than BPI. ${ }^{6}$ BPII adopts a significantly different pathway to recovery after a strong electric field is switched off. It achieves complete restoration, whereas BPI remains trapped in metastable configurations. ${ }^{7}$

In this letter, we present an experimental observation showing that the two phases also differ in their response to alignment layers (ALs). This difference facilitates a convenient

${ }^{a}$ Electronic mail: pankaj.joshi@elis.ugent.be pathway for preparation of samples with large domains of BPI and BPII without going through tedious multiple thermal cycling.

A BPLC mixture was prepared using nematic liquid crystals JC1041-XX (48.18 wt. \%, from Chisso; $\Delta \mathrm{n}=0.142$, $\Delta \varepsilon=5.7$ at $25^{\circ} \mathrm{C}^{8}$ ) and $5 \mathrm{CB}$ (38.08wt. \%, from Synthon Chemicals; $\Delta \mathrm{n}=0.19, \Delta \varepsilon=11$ at $25^{\circ} \mathrm{C}^{8}$ ), chiral dopant R5011 (3.2 wt. \%, from HCCH), monomer EHA (3.43 wt. \%, from Synthon Chemicals), cross-linker RM257 (6.66 wt. \%, form Merck), and photoinitiator DMPAP (0.52 wt. \%, from Merck). Three types of LC cells, listed in Table I, were filled with this LC mixture at room temperature.

The cells of $9 \mu \mathrm{m}$ thickness are from Instec, whereas the other types are assembled in our laboratory. $\mathrm{SiO}_{2}$ layer is $6 \mathrm{~nm}$ thick and evaporated at an angle of $45^{\circ}$ with respect to the substrate normal. Both types of alignment layers induce homogeneous alignment in the nematic liquid crystals used in this study. A staggered cooling/heating scheme with a rate of $0.1^{\circ} \mathrm{C} / \mathrm{min}$ and $1 \mathrm{~min}$ hold at every tenth of a degree Celsius was employed in the BP range. The in-situ polymerization was done with a UV source at $365 \mathrm{~nm}$ with a total energy dose of $200 \mathrm{~mJ} / \mathrm{cm}^{2}$ administered at an irradiance of $50 \mathrm{~mW} / \mathrm{cm}^{2}$.

Transmission spectra were recorded using a spectrometer (USB2000, Ocean Optics for visible and LAMBDA35, PerkinElmer for UV) with samples held at constant temperature on a heating stage (TS-4MP, Physitemp). In order to minimize noise, spectra at every temperature were measured five times and subsequently averaged. Transmittances were normalized with respect to the transmission of the BP cell in the isotropic state.

TABLE I. Details of different types of liquid crystal cells used.

\begin{tabular}{lc}
\hline Type 1 & $9 \mu \mathrm{m}$ thickness, rubbed polyimide antiparallel alignment layers \\
Type 2 & $10 \mu \mathrm{m}$ thickness with obliquely evaporated \\
& $\mathrm{SiO}_{2}$ antiparallel alignment layers \\
Type 3 & $10 \mu \mathrm{m}$ thickness without alignment layers \\
\hline
\end{tabular}




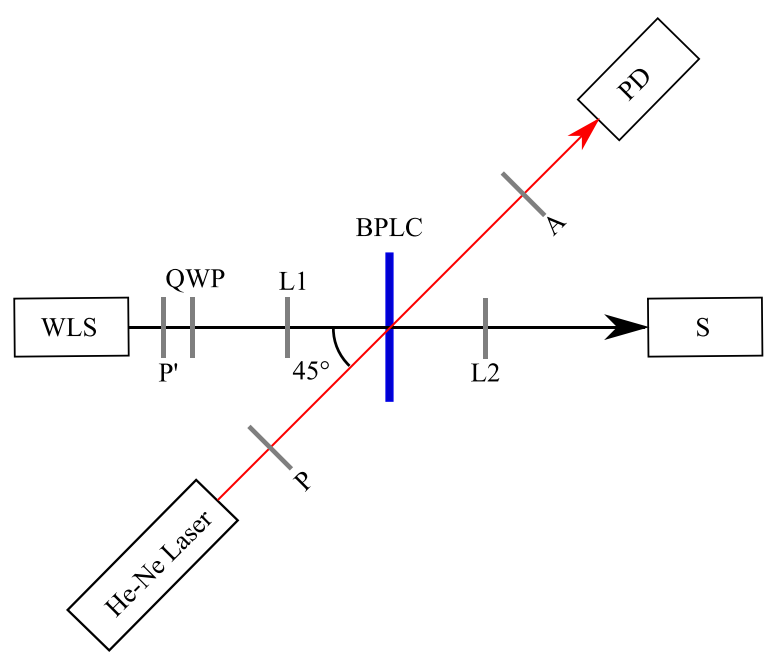

FIG. 1. Experimental setup for the measurement of switching speeds and transmission spectra. WLS is the broadband white light source; $\mathrm{P}^{\prime}$ is a polarizer; QWP is a quarter wave plate; $\mathrm{P}\left(+45^{\circ}\right)$ and $\mathrm{A}\left(-45^{\circ}\right)$ are polarizer and analyzer, respectively; PD is a photodiode; $\mathrm{S}$ is the spectrometer; and L1 and $\mathrm{L} 2$ are focusing lenses.

Switching speed measurements were done using a red $\mathrm{He}-\mathrm{Ne}$ laser at $633 \mathrm{~nm}$ and an avalanche photo-diode (C 5460-01, Hamamatsu). The measurement setup is shown in Figure 1. The axis of the polarizer $\mathrm{P}^{\prime}$ makes an angle of $45^{\circ}$ with the optical axis of the quarter wave plate (QWP) in order to get circularly polarized light of the same handedness as that of the cell under test.

In order to carefully identify the phase transitions, the evolution of the peak Bragg reflection $\left(\lambda_{\mathrm{o}}\right)$ wavelength with temperature was recorded. We first look at the heating cycle. Figure 2(a) shows that the peak Bragg wavelength remains within $\sim 5 \mathrm{~nm}$ of its value at $30^{\circ} \mathrm{C}$ as the temperature is increased to $43.9^{\circ} \mathrm{C}$. The Bragg reflection in this region is due to the presence of a chiral nematic $\left(\mathrm{N}^{*}\right)$ phase. The first discontinuity in $\lambda_{\mathrm{o}}$ at $44{ }^{\circ} \mathrm{C}$ is caused by the broadening in Bragg reflection in the $\mathrm{N}^{*}$ phase as can be seen from Figure 2(b). Such broadening might be caused by the stacking of two of more layers of $\mathrm{N}^{*}$ phases reflecting at slightly

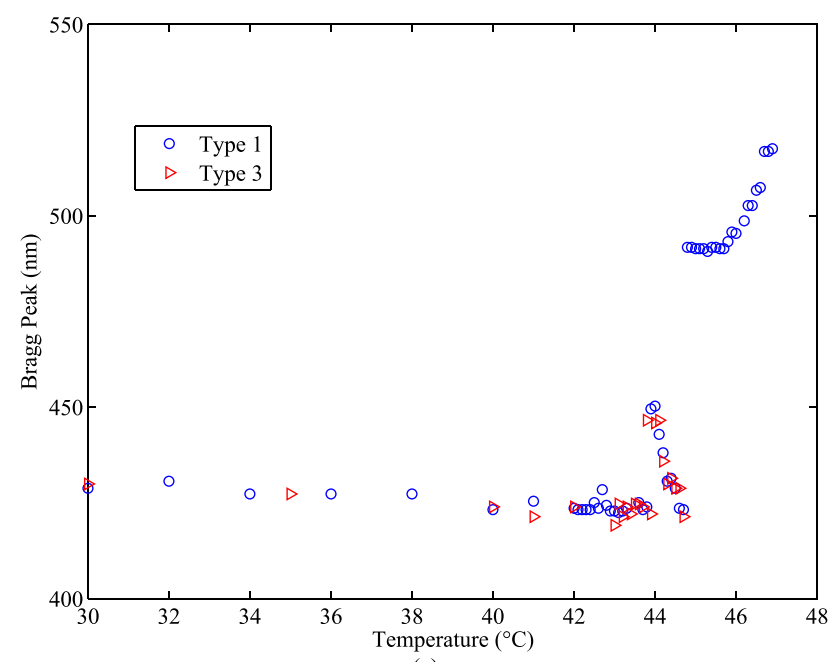

(a)

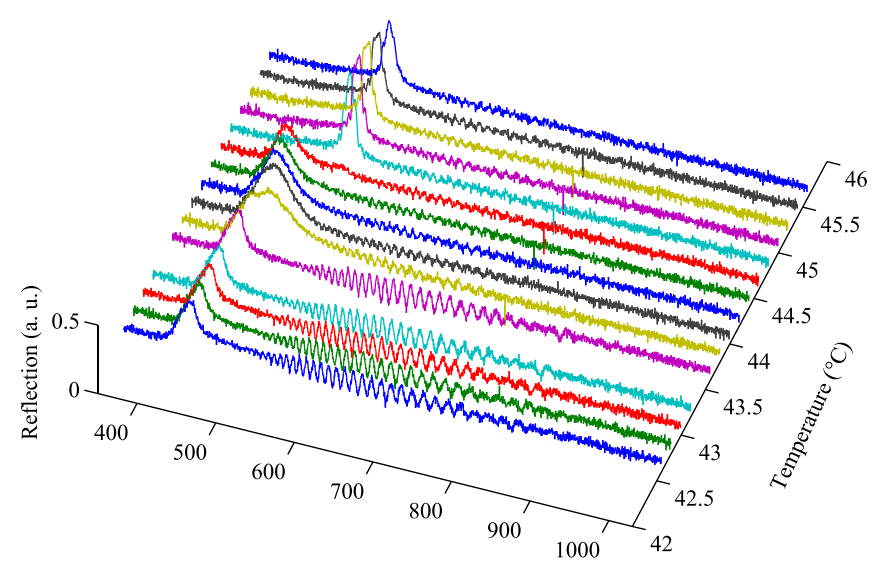

Wavelength (nm)

(b)

FIG. 2. (a) Evolution of the peak Bragg reflection wavelength with increasing temperature. Blue circles are for cells with alignment layer and red triangles for cells without alignment layer. (b) Spectra of type 1 at temperatures in the region of jumps in peak Bragg wavelength.

different adjacent wavelengths in the cell, on top of each other. The second discontinuity in $\lambda_{\mathrm{o}}$ of more than $70 \mathrm{~nm}$ at $44.7^{\circ} \mathrm{C}$ is due to the phase transition from $\mathrm{N}^{*}$ to BP. No such
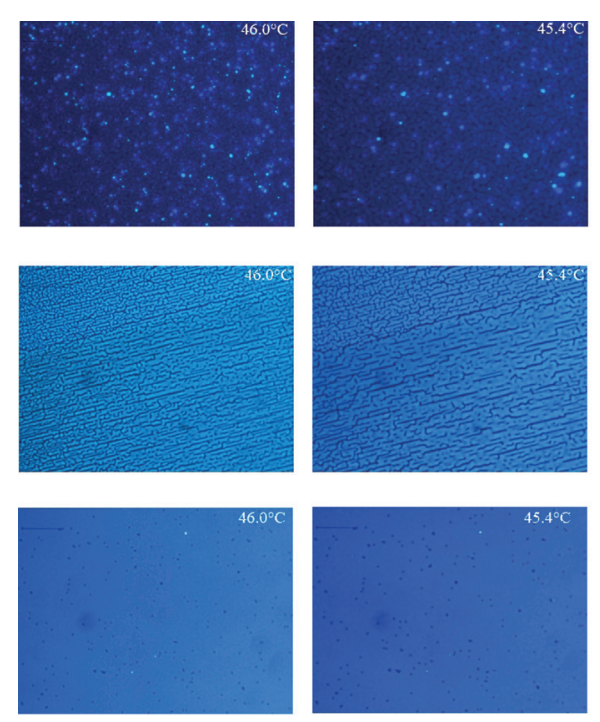
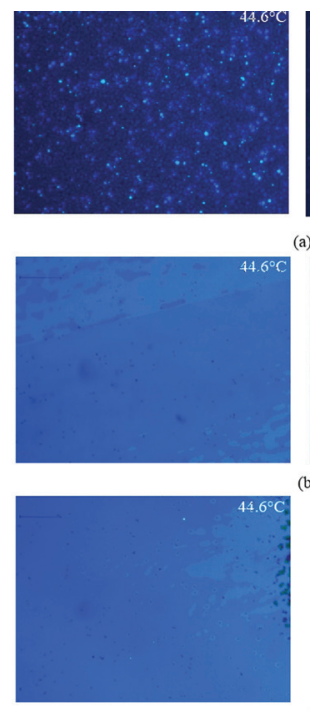
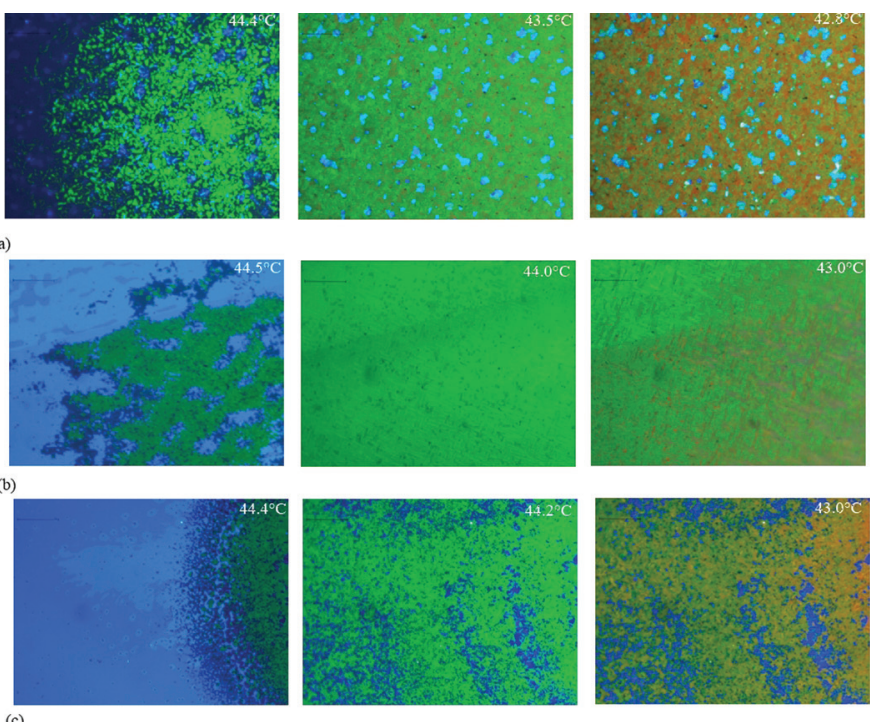

FIG. 3. POM of textures at different temperatures (a) type-3, (b) type-1, and (c) type-2. 


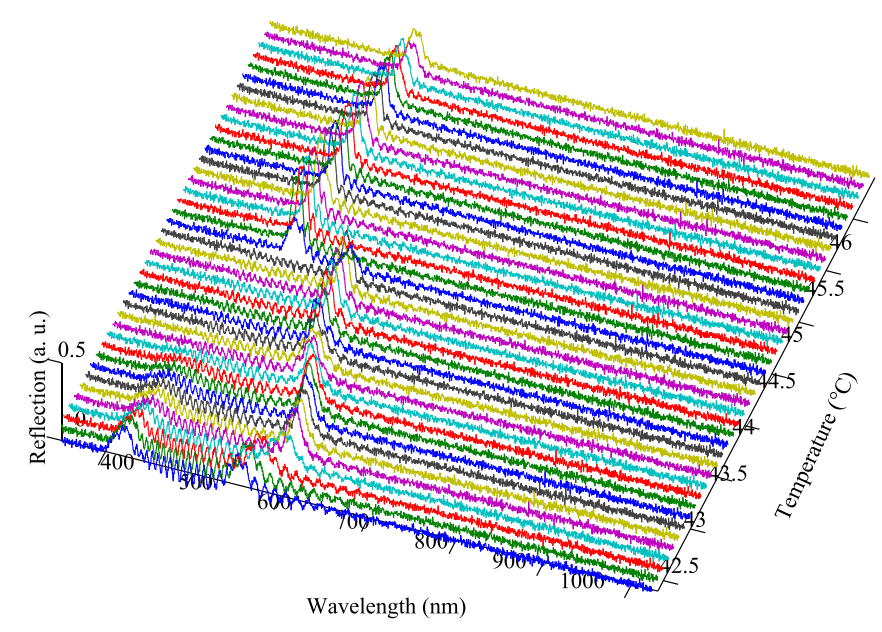

FIG. 4. Transmission versus wavelength and temperature for a type-1 cell. The results for type- 2 cells are very similar. The discontinuity in the peak Bragg reflection wavelength at $44.5^{\circ} \mathrm{C}$ indicates the phase change from BPII to BPI.

discontinuity was found in the cell without AL, although the broadening of the reflection peak coincided with that of the cell with ALs, as can be seen from Figure 2(a).

In the cell without alignment layers, flow generated by capillary filling induces an alignment which is destroyed after the first heating to the isotropic state. Because of this, the evolution of $\lambda_{0}$ in the $\mathrm{N}^{*}$ phase of cells without AL can only be studied in the first heating cycle. However, it was observed that a more uniform texture of BP is obtained in the cooling cycle. Therefore, in the remainder of the text, all results are obtained in the cooling cycle.

Figure 3 shows the polarization optics micrographs (POMs) of the three types of samples arranged as temperature decreases in the cooling cycle within the BP existence range. The POMs in the first three columns of Figure 3 show a clear distinction between cells with AL and cells without AL. In type-3 cells, without ALs (Figure 3(a)), small scattered bright domains can be seen to appear below clearing temperature, whereas in type-1 (Figure 3(b)) and type-2 (Figure 3(c)) cells, with ALs, very different and uniform textures are present. In the cell with rubbed PI ALs, small domains of BP merge along the rubbing direction first. As the temperatures are lowered, further domains merge in the lateral direction also, forming textures of higher uniformity, see Figure 3(b) for textures at $46.0^{\circ} \mathrm{C}, 45.4{ }^{\circ} \mathrm{C}$, and $44.6^{\circ} \mathrm{C}$. The topological defects on the poly-imide (PI) layer created by the rubbing process ${ }^{9}$ are most likely the cause of this behavior. As the process of oblique evaporation of $\mathrm{SiO}_{2}$ does not create such topological defects, these lines were absent in type-2 cells (Figure 1(b)), confirming our suspicion. On further lowering of the temperature, a distinct change in texture, indicating a phase transition, occurring around $44.5^{\circ} \mathrm{C}$ can be clearly seen in Figure 3 in all three types of samples.

For temperatures below $44.5^{\circ} \mathrm{C}$, the characteristic platelets of BP are present, as can be seen from the last three columns of Figure 3. To understand the nature of the phase present above $44.5^{\circ} \mathrm{C}$, we analyzed the respective transmission spectra. In type- 3 cells, without $\mathrm{AL}$, no Bragg reflection in the visible range was found to be present for temperatures above $44.5^{\circ} \mathrm{C}$, whereas the cells with ALs possessed well defined Bragg reflection in the visible range at those temperatures. However, these cells do show platelet texture which is indicative of BP, e.g., reflecting in the UV region.

Since the phase transition in BPLCs is weakly first-order in nature, a discontinuity in the peak Bragg-reflection wavelength versus temperature is a good indicator of phase transition. ${ }^{10,11}$ Such a graph is shown in Figure 4 for type-1. A

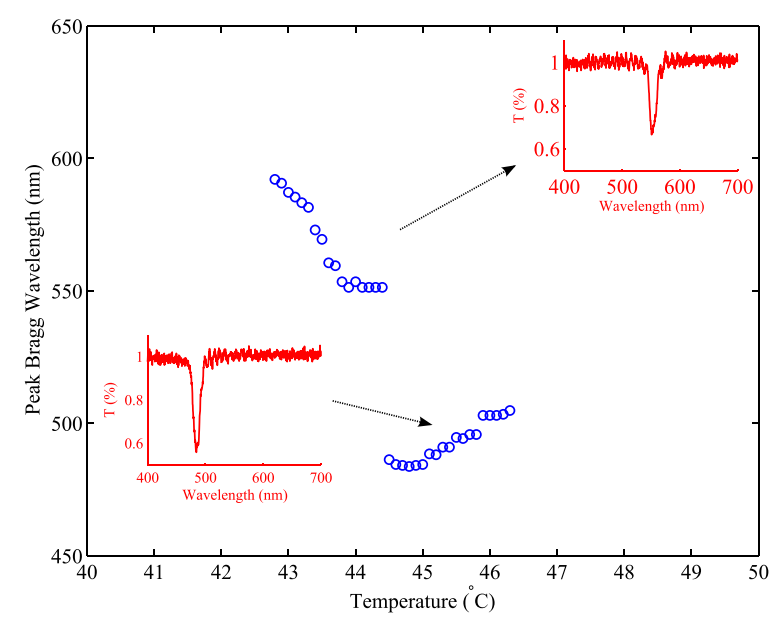

(a)

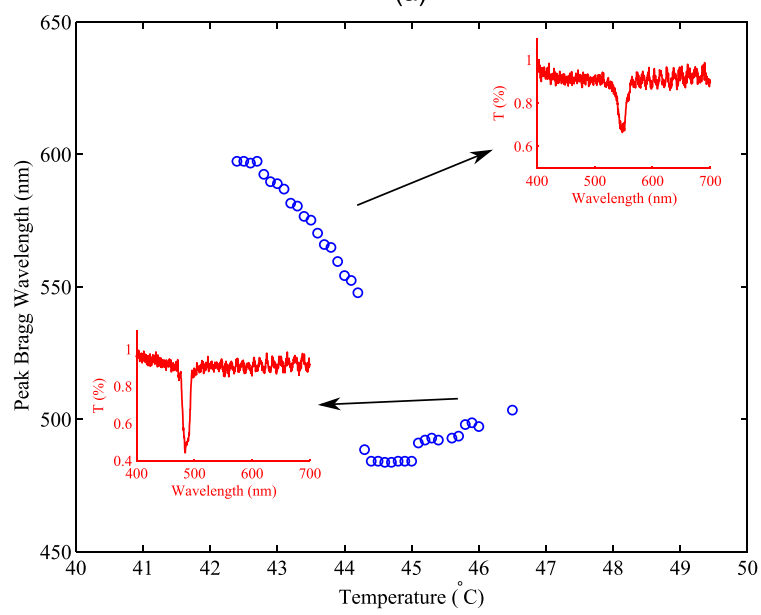

(b)

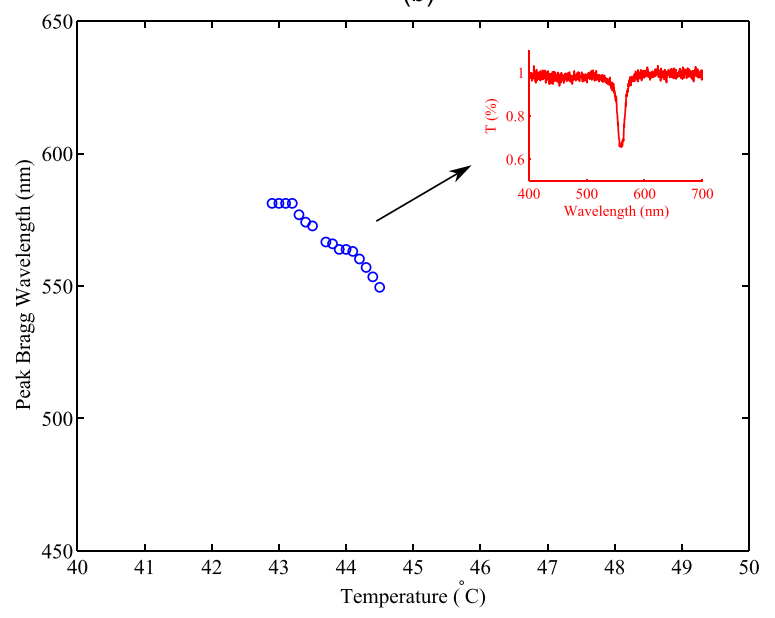

(c)

FIG. 5. Variation of peak Bragg reflection wavelength as a function of temperature for cells of (a) type-1, (b) type-2, and (c) type-3. Inset presents a single transmission spectrum belonging to the respective phase. 


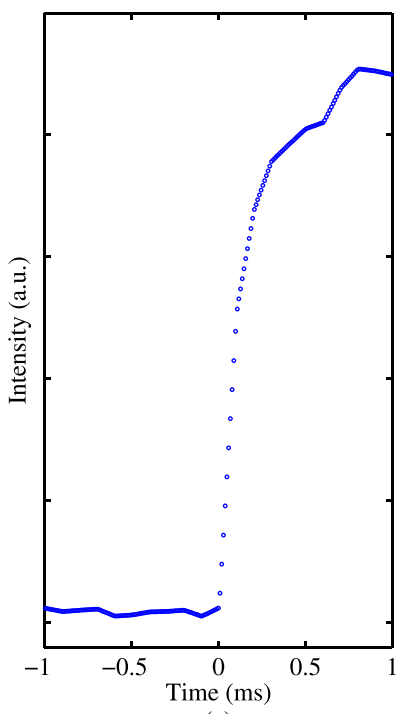

(a)

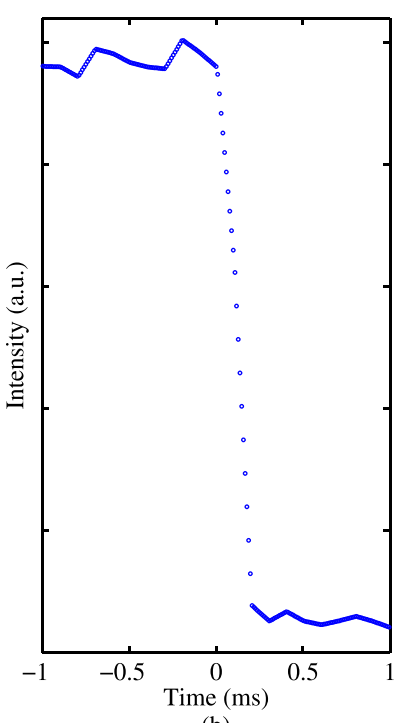

(b)
FIG. 6. Switching speeds of blue phase II liquid crystals.

discontinuity of $\sim 65 \mathrm{~nm}$ in $\lambda_{0}$ is consistent with previously reported values involving phase transition from BPII to BPI. ${ }^{6,12}$ Smaller peaks around $440 \mathrm{~nm}$ below $42.7^{\circ} \mathrm{C}$ correspond to a $\mathrm{N}^{*}$ phase. This is a first indication that the two phases present in the cells with ALs may be BPI and BPII.

Alignment layers have previously been reported to induce uniform domains of BPs; ${ }^{13,14}$ however, to the best of our knowledge, their preferential influence on one of two cubic BPs has never been documented. It is also worth noting that we did not observe the previously reported pinning effect where alignment layers substantially suppress the change in photonic bandgap in response to changes in temperature. ${ }^{15}$

Figure 5 shows the evolution of $\lambda_{0}$ for the three types of cells in the cooling cycle. The corresponding insets show one transmission spectrum belonging to each phase. The maximum reflection observed above discontinuity temperatures is larger than the one below discontinuity temperature. It takes a certain number of pitches to be present before the Bragg's reflection establishes. BPI has a larger lattice constant than BPII, which entails that for a given cell gap fewer pitches will be present, which implies weaker reflection. This further strengthens our belief that the phases are BPI and BPII, respectively.

To provide further evidence supporting that the phase which is present in samples with alignment layers and also possesses Bragg reflection is $\mathrm{BP}$ and not conventional $\mathrm{N}^{*}$, we carried out switching speed measurements. A He-Ne laser at $632 \mathrm{~nm}$ was used as this wavelength lies just outside the Bragg reflections region of BP used in this study. Switching speeds and transmission spectra were measured simultaneously. The results for the phase present above $44.5^{\circ} \mathrm{C}$ in type- 1 cell are shown in Figure 6 . The switching on and off times are $364 \mu \mathrm{s}$ and $167 \mu \mathrm{s}$, respectively. The switching speeds in type- 2 cells are also sub-millisecond.

Having established the phase present in cells with $\mathrm{AL}$ above $44.5^{\circ} \mathrm{C}$ as BPII, we looked at the transmission of type- 3 cells above this temperature, shown in Figure 7. A dip around $338.5 \mathrm{~nm}$ indicates that BPII domains are indeed reflecting in UV. With AL, peak of Bragg reflection shifts to $486.3 \mathrm{~nm}$.

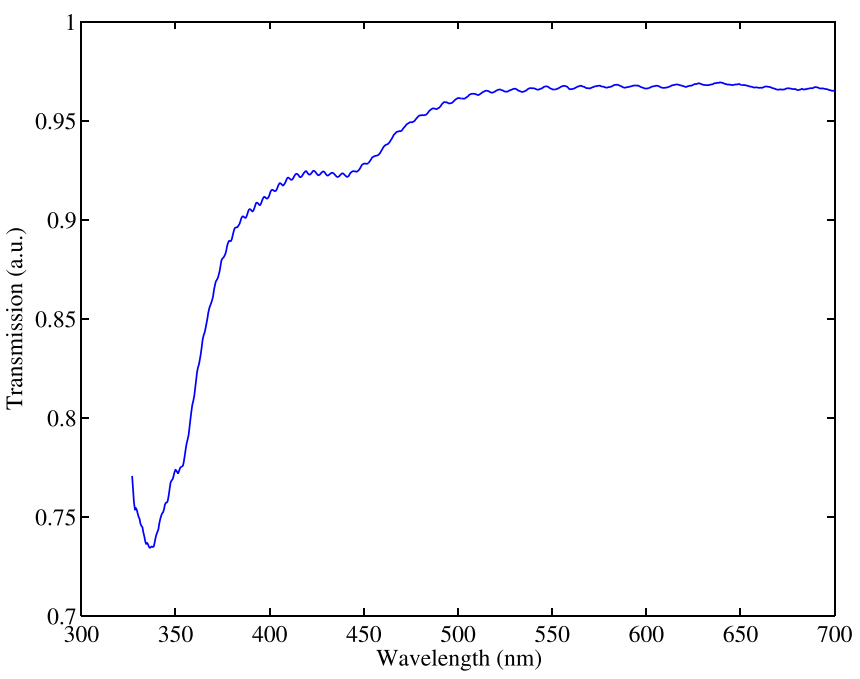

FIG. 7. Transmission spectrum of type-3 cell at temperature above transition.

Based on the above presented series of observations, it can be concluded that homogeneous alignment layers selectively influence BPII domains by reorienting and assisting them to form large domains, whereas this does not occur for BPI. We were also able to polymer stabilize the desired phases, which establishes the potential of this method in assisting selective studies of the two phases.

This work was partly supported by the Research Foundation-Flanders (FWO) through the project No. 3GA04711 ("Study of liquid crystals dispersed in microstructured elastic host materials") and partly by the IWT through the SBO project SECONDOS, IWT-nr 120019, 20132016. We would also like to thank Professor Kristiaan Neyts and Ms. Gaby Nordendorf for their fruitful comments, JNC Corporation, Japan for providing us with the liquid crystal mixture (No. JC1041-XX) and Oliver Willekens for providing us with PI LC cells and his assistance with UV-Vis spectrometer.

${ }^{1}$ L. Rao, J. Yan, S. T. Wu, S. I. Yamamoto, and Y. Haseba, Appl. Phys. Lett. 98, 081109 (2011).

${ }^{2}$ Y. Hisakado, H. Kikuchi, T. Nagamura, and T. Kajiyama, Adv. Mater. 17, 96 (2005).

${ }^{3}$ Y. Chen, J. Yan, J. Sun, S. T. Wu, X. Liang, S. H. Liu, and J. W. Shiu, Appl. Phys. Lett. 99, 201105 (2011).

${ }^{4}$ H. Kikuchi, M. Yokota, Y. Hisakado, H. Yang, and T. Kajiyama, Nat. Mater. 1, 64 (2002).

${ }^{5}$ O. Henrich, K. Stratford, M. E. Cates, and D. Marenduzzo, Phys. Rev. Lett. 106, 107801 (2011).

${ }^{6}$ H. Choi, H. Higuchi, and H. Kikuchi, Appl. Phys. Lett. 98, 131905 (2011).

${ }^{7}$ A. Tiribocchi, G. Gonnella, D. Marenduzzo, and E. Orlandini, Soft Matter 7, 3295 (2011).

${ }^{8}$ S. Shibayama, H. Higuchi, Y. Okumura, and H. Kikuchi, Adv. Funct. Mater. 23, 2387 (2013).

${ }^{9}$ L. T. Creagh and A. R. Kmetz, Mol. Cryst. Liq. Cryst. 24(1-2), 59 (1973).

${ }^{10}$ D. C. Wright and N. D. Mermin, Rev. Mod. Phys. 61(2), 385 (1989).

${ }^{11}$ J. H. Flack and P. P. Crooker, Phys. Lett. A 82(5), 247-250 (1981).

${ }^{12}$ H. J. Coles and M. N. Pivnenko, Nature 436(7053), 997 (2005).

${ }^{13}$ P. Nayek, H. Jeong, H. R. Park, S. W. Kang, S. H. Lee, H. S. Park, and H. S. Kim, Appl. Phys. Express 5(5), 051701 (2012).

${ }^{14}$ J. Yan, S. T. Wu, K. L. Cheng, and J. W. Shiu, Appl. Phys. Lett. 102(8), 081102 (2013).

${ }^{15}$ H. Y. Liu, C. T. Wang, C. Y. Hsu, and T. H. Lin, Appl. Opt. 50(11), 1606 (2011). 